\title{
Evaluación de las deformaciones y su pronóstico en el Turbo Generador 5, de la planta termo-energética de Nuevitas, Camagüey
}

\section{(Deformations evaluations and forecast in Turbo Generator 5 in the thermic-energetic plant of Nuevitas, Camagüey)}

\author{
Michael Álvarez González¹, Vladimir Mederos Pérez², Miguel Ángel Hernández Machado³ \\ y Rafael Matamoros García ${ }^{4}$
}

\begin{abstract}
Resumen
La evaluación, el análisis y pronóstico del comportamiento de las deformaciones en el Turbo Generador 5, de la planta termo-energética de Nuevitas, fue el objetivo central de esta investigación. Para ello fueron realizadas mediciones geodésicas de control de asentamiento, cronológicamente distribuidas en el tiempo a marcas específicas en la estructura. Estos datos, junto a la modelación físico-numérica por medio del Método de los Elementos Finito, permitió evaluar el proceso de interacción carga-deformación e interacción suelo estructura. Luego de lograr un registro histórico del comportamiento tenso-deformacional plano, se realizó un pronóstico de las deformaciones en la losa de apoyo por medio de Modelos Arima, para evaluar a corto plazo las desalineaciones futuras permisibles, registradas sistemáticamente en los ejes de los rotores del sistema mecánico del turbo generador.
\end{abstract}

\section{Palabras clave}

Geodesia, carga-deformación, interacción suelo estructura, método de los elementos finitos, modelos Arima.

\begin{abstract}
The evaluation, analysis and deformations forecast in the Turbo Generator 5 of the water heater-energy plant of Nuevitas, it was the main objective of this research. For they were carried out it chronologically geodesic surveying to establishment control record distributed in same time period to specific marks along the structure. These data were used in the physical-numeric models, by Finite Elements Method, it allowed evaluate the load-deformation process and floor-structures interaction. After achieving the historical record of stress-strain plane state, forecast deformations was proposing for the support structure by Arima's models to evaluate in short term the not-alienations permissible future registered systematically in the axes of the mechanical system rotors in the plant.
\end{abstract}

\section{Keywords}

Geodesy, load-deformation, finite element method, soil-structure interaction, Arima model

\section{Introducción}

El estudio y control de las deformaciones en obras de ingeniería, implica una garantía de seguridad durante el proceso de puesta en marcha, explotación y mantenimiento operativo. Este elemento recobra mayor relevancia aun cuando se trata de elementos de la geodesia ingeniera, al considerar que las precisiones requeridas rondan sobre las unidades y hasta fracciones del milímetro.

En plantas de generación energética a partir de turbo generadores por combustión, generalmente se crea una amplia red de control de las deformaciones; tanto a toda la estructura

1 Empresa de Investigaciones y Proyectos Hidráulicos de Villa Clara (IPH VC), Santa Clara. Villa Clara, Cuba. [michael@ vc.hidro.cu), https://orcid.org/0000-0003-2953-4762]

2 Grupo Empresarial Geocuba Villa Clara - Sancti Spíritus, Santa Clara. Villa Clara, Cuba. [vmederos@vcl.geocuba.cu), https:// orcid.org/0000-0001-6043-3834]

3 Universidad Central "Marta Abreu" de Las Villas (UCLV), Santa Clara. Villa Clara. Cuba. [mihmachado@uclv.cu]

$4 \quad$ Universidad Central "Marta Abreu" de Las Villas (UCLV), Santa Clara. Villa Clara, Cuba. [rmatamoros@uclv.cu] 
de soporte como al terreno circundante. En el presente trabajo se exponen los resultados alcanzados durante una serie de trabajos de control geométrico a partir de una serie de fallas y reparaciones costosas, que fueron realizadas a la planta, comenzando con la desalineación sistemática de los ejes y rotores del Turbo Generador 5 (TG5) de la Termo Eléctrica "10 de octubre" en Nuevitas, Camagüey (véase la figura 1).

Figura 1. Ubicación general de la Termo Eléctrica "10 de octubre" en Nuevitas, Camagüey.

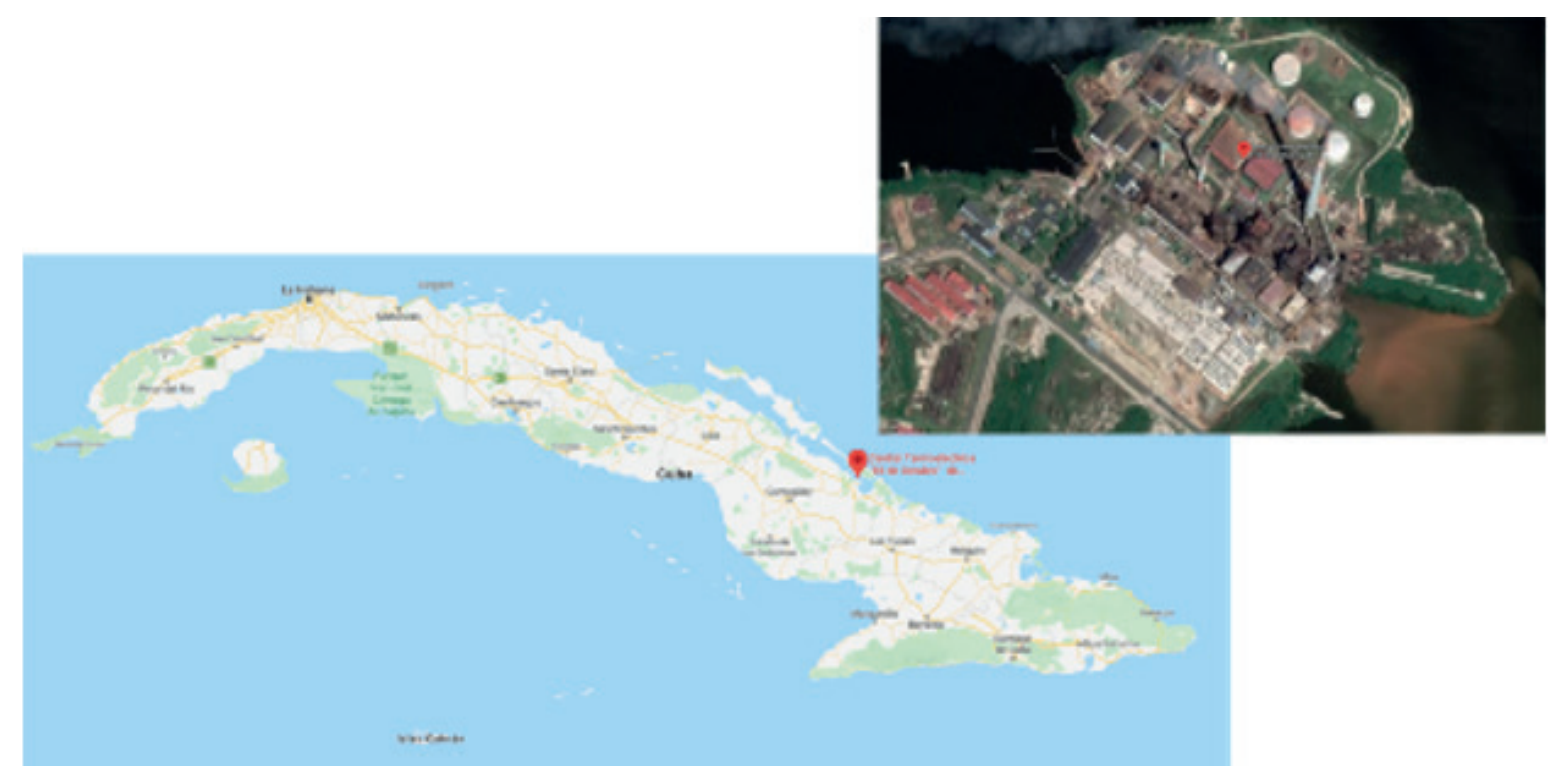

Fuente: Google Earth

Este trabajo tuvo por objetivo realizar un diagnóstico ingeniero geodésico, su posterior evaluación y pronóstico de las deformaciones, por medio de la modelación físico-numérica con el Método de los Elementos Finitos (MEF) del Turbo Generador 5 (TG5), en su interacción con las cargas actuantes entre la losa soportante y el suelo de la Termo Eléctrica de Nuevitas en la ciudad de Camagüey.

Las bases teóricas sobre las cuales se fundamentó esta investigación, se justifican a partir de varios estudios publicados sobre las deformaciones en obras ingenieras (Álvarez, 2018; Blanco, Pacheco \& Moreno, 2020; Delgado, 2011; Hernández, 2012; Kopáčik, Erdélyi \& Kyrinovič, 2020a, 2020b). Para lograr este objetivo se propone la ejecución de los procesos investigativos, acorde a la filosofía de análisis propuesta en la figura 2, en el que se realiza un primer momento en la definición de las secciones transversales y perfiles geológicos, correspondientes a la distribución espacial, conforme a la influencia y alcance que se pretende abarcar en el presente diagnóstico. Además de establecer los parámetros físico-mecánicos de los materiales utilizados, tanto en la construcción como en el suelo donde se encuentra el TG5.

El esquema propuesto se basa en las experiencias previas por parte de investigadores de la Universidad Central Marta Abreu de Las Villas, contenidas en diversos campos de la ingeniería (L. Álvarez, 1998; Álvarez, 2018; Quevedo, 2001a, 2001b; Recarey, 1999). 
Figura 2. Esquema general de trabajo para la simulación, evaluación y diagnóstico de las deformaciones en el TG5
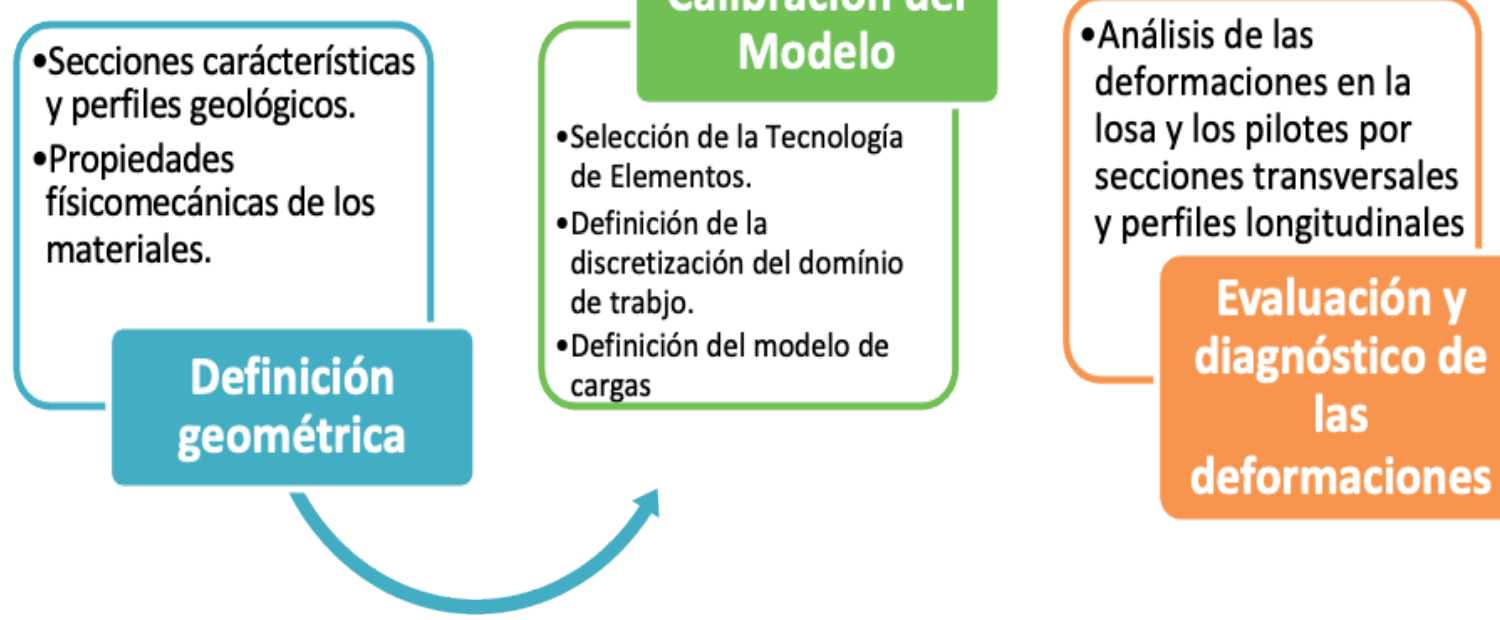

En un segundo momento se desarrolló un proceso de calibración del modelo, en el que se definió la Tecnología de Elementos Finitos (TEF), a utilizar según la geometría del dominio de trabajo, en función del proceso de discretización o subdivisión de la malla utilizada en el modelo conceptual. Ello permitió arribar a un tercer momento, con una comparación de la curva de presión ejercida por la estructura al suelo y un chequeo del comportamiento del trabajo de los pilotes y la losa portante de la estructura.

\section{Metodología}

\section{Selección y definición geométrica de las secciones transversales y perfiles geológicos}

En la figura 3 se representan los dos ejes principales, acorde a la distribución espacial de la losa y los pilotes, acotadas todas las dimensiones en unidades métricas (m).

En la figura 4 son representadas las vistas en corte por los ejes izquierdo y derecho, con el acople de los perfiles geológicos, según las capas identificadas por las calas, realizadas en los datos previos suministrados por el cliente.

En el proceso de la conformación y definición de las secciones geométricas se decidió realizar los análisis de modelación, por medio de los ejes izquierdo (estos son denominados con el símbolo [']) y derecho, según se identifica en la figura 4, que representa el ensamblaje de los modelos, por ser los más representativos del trabajo tenso-deformacional de la losa, a todo lo largo y no en pequeñas secciones transversales, lo cual es muy útil en el caso de que se pretenda realizar estudios más localizados, si es que se identifica algún comportamiento de anomalías (fisuras o grietas, zona de acumulación de tenciones, etc.). 
Figura 3. Esquema general de las dimensiones principales y ejes del TG5

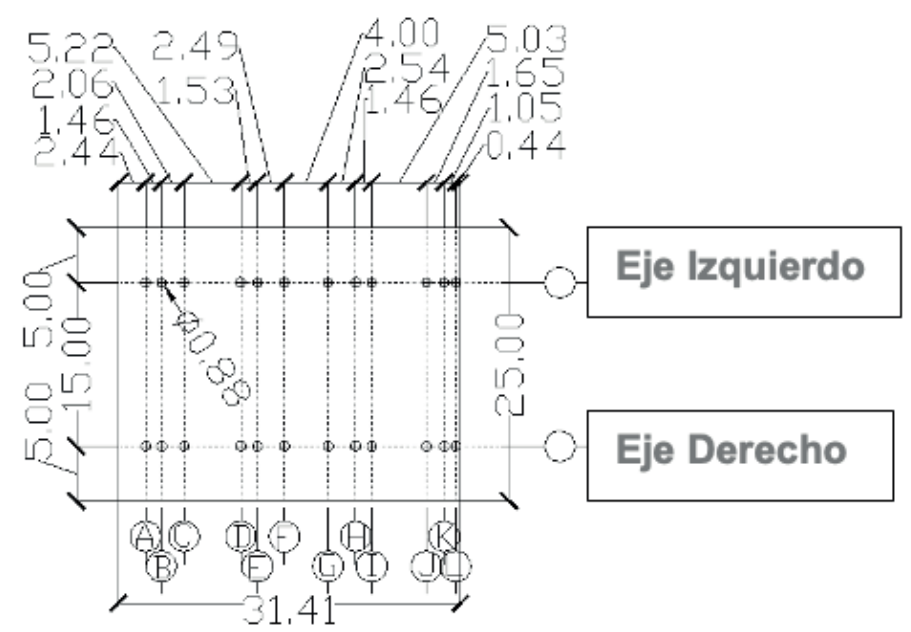

Figura 4. Esquema general de los perfiles de trabajo por los ejes izquierdo y derecho en el TG5

Eje Izquierdo del TG5

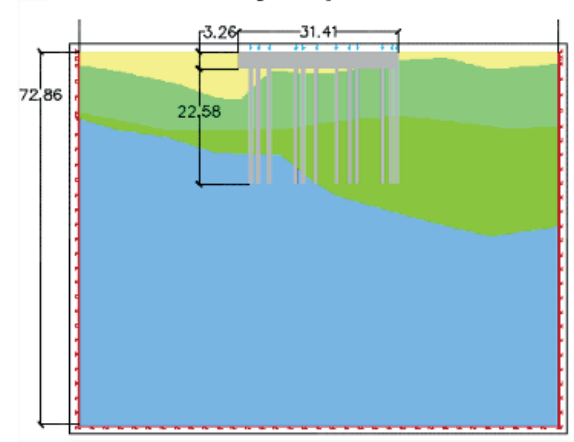

Eje Derecho del TG5

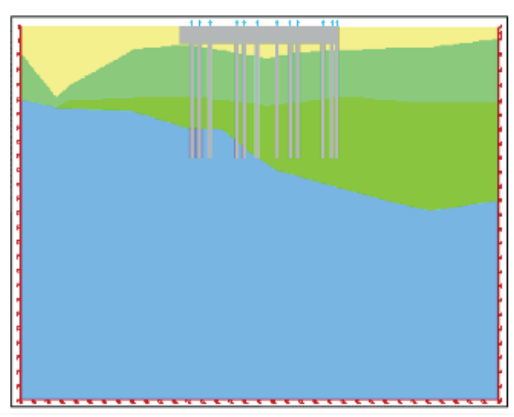

Leyenda

Hormigón

Capa Goolágica A

Capa Geológica B

Capa Goológica C

Capa Goolágica D a más

de $25 \mathrm{~m}$ de profundidad

Condiciones de bordes

impuestas al modelo

Condición de borde por

peso de las máquinas

Además, se anexa a continuación la tabla 1, en la que se identifican las capas geológicas y los parámetros físico-mecánicos, utilizados en el proceso de modelación de los materiales del suelo y del hormigón, según se muestra en la figura 5.

Tabla 1. Parámetros físico-mecánicos utilizados en el proceso de modelación físico-numérica de los materiales, en los modelos correspondientes a los ejes izquierdo y derecho

\begin{tabular}{|c|c|c|c|c|c|c|}
\hline \multirow{2}{*}{ Parámetros } & \multicolumn{7}{|c|}{ Modelos/Materiales } \\
\cline { 2 - 7 } & $\begin{array}{c}\text { Lineal } \\
\text { Elástico }\end{array}$ & $\begin{array}{c}\text { Elasto- } \\
\text { Plástico }\end{array}$ & $\begin{array}{c}\text { Elasto- } \\
\text { Plástico }\end{array}$ & $\begin{array}{c}\text { Elasto- } \\
\text { Plástico }\end{array}$ & $\begin{array}{c}\text { Elasto- } \\
\text { Plástico }\end{array}$ & $\begin{array}{c}\text { Elasto- } \\
\text { Plástico }\end{array}$ \\
\cline { 2 - 7 } & Hormigón & CGA & CGB & CGC & CGD & CGD+25 m \\
\hline Módulo de Young $(E)(\mathrm{kPa})$ & 28000000 & 15000 & 14000 & 21000 & 23000 & 38000 \\
\hline Peso unitario $\left(\mathrm{kn} / \mathrm{m}^{3}\right)$ & 2.5 & 2.7 & 2.75 & 2.77 & 2.72 & 2.71 \\
\hline Módulo de Poisson & 0.3 & 0.49 & 0.49 & 0.49 & 0.49 & 0.49 \\
\hline Módulo de cohesión $(\mathrm{kPa})$ & - & 90 & 97 & 124 & 144 & 360 \\
\hline Ángulo de fricción $\left(^{\circ}\right)$ & - & 8 & 5 & 1 & 9 & 5 \\
\hline Ángulo de dilatación $\left(^{\circ}\right)$ & - & 8 & 5 & 1 & 9 & 5 \\
\hline
\end{tabular}


Figura 5. Representación del comportamiento de un modelo elasto-plástico, de los materiales del suelo

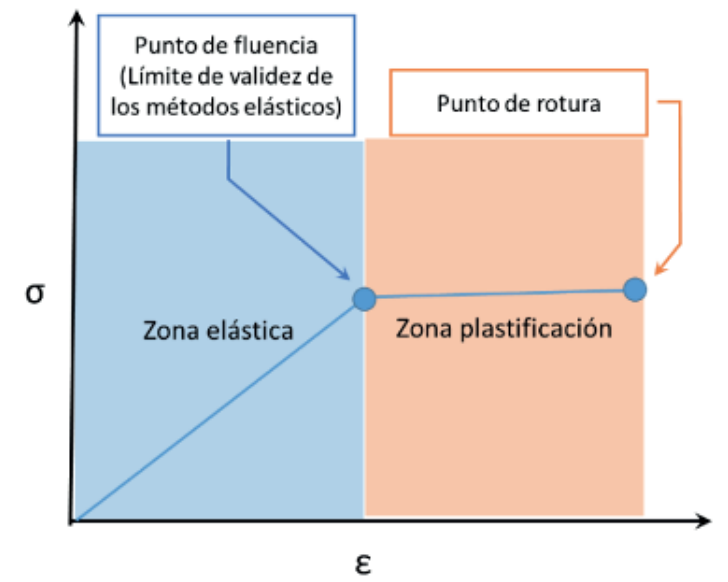

Fuente: (Álvarez, 2018: 107)

En la tabla 2 se listan las cargas concentradas al realizar una distribución físico-geométrica de las masas correspondientes al equipamiento tecnológico colocado sobre la losa. Se calculó la carga correspondiente, que se generaba sobre cada pilote desde la superficie de la losa soportante.

Tabla 2. Cargas concentradas asignadas a la losa soportante geométricamente sobre los pilotes

\begin{tabular}{|c|c|c|c|}
\hline No. Pilote & Carga concentrada (kN) & No. Pilote & Carga concentrada (kN) \\
\hline $1-1^{\prime}$ & 1392.90 & $7-7^{\prime}$ & 1076.40 \\
\hline $2-2^{\prime}$ & 1366.20 & 8-8' & 1029.80 \\
\hline 3-3' & 1327.70 & 9-9' & 1084.00 \\
\hline $4-4^{\prime}$ & 1227.60 & $10-10^{\prime}$ & 906.37 \\
\hline $5-5^{\prime}$ & 1199.90 & $11-11$ & 875.84 \\
\hline $6-6^{\prime}$ & 1152.00 & $12-12^{\prime}$ & 855.35 \\
\hline
\end{tabular}

Para el proceso de selección de la Tecnología de Elementos Finitos (TEF), a utilizar en la modelación como proceso de calibración numérica del modelo. En el proceso de selección de la forma geométrica del TEF fue descartado el uso del elemento rectángulo, por no ajustarse correctamente a zonas en la distribución geométrica y calcular un error en la plataforma utilizada, que no se ajusta a la malla con los requisitos previamente determinados por el autor durante el proceso de selección de la TEF (figura 6).

Se realizó la siguiente propuesta para simplificar la nomenclatura de la TEF:

- DimTE1-am $\rightarrow$ Malla de rectángulos y triángulos con nodos auxiliares, donde a es la dimensión global de cada elemento en el proceso de iteración.

- DimTE2-@m $\rightarrow$ Malla de triángulos sin nodos auxiliares, donde @ es la dimensión global de cada elemento en el proceso de iteración. 
Figura 6. Procedimiento para la obtención de la densidad de malla óptima utilizada, para un tipo de elemento finito

Se obtiene para cada TEF (i):

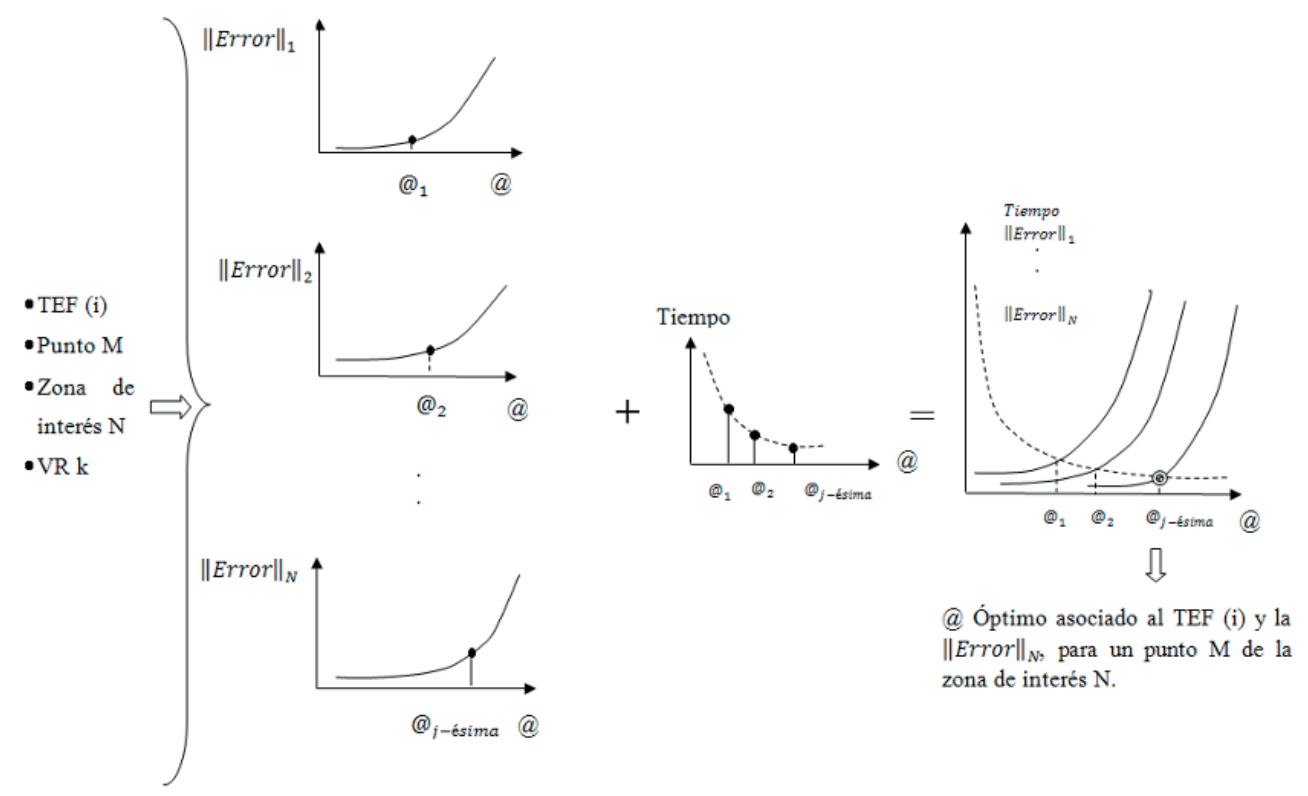

Fuente: (Álvarez, Mesa, \& Recarey, 2012)]

\section{Resultados}

\section{Resultados obtenidos empleando el Método de los Elementos Finitos (MEF), esquema de análisis de modelación y datos iniciales}

En este acápite se procede a realizar una estrategia de evaluación de los resultados alcanzados por los modelos físico-matemático-estadísticos, empleados para determinar el comportamiento histórico de las deformaciones, registradas por métodos geodésicos en la losa soportante del Turbo Generador 5.

Acorde a lo anteriormente planteado, se decidió asumir la estrategia de modelación (véase figura 7), en la que se determinó el proceso de redistribución de las tensiones, para el comportamiento de la sección típica correspondiente al área de estudio segmentada en dos secciones longitudinales principales, para abarcar todo el largo de la losa, pasando por los centros de los pilotes.

Figura 7. Representación gráfica de la estrategia de modelación a seguir durante la simulación con el Método de los Elementos Finitos

$\mathrm{TI}_{0}$ • Modelación de la redistribución de las tensiones iniciales de la estructura.

C/D - Simulación de las relación tenso-deformacional de la estructura a las cargas.

Ft Se realiza el pronóstico futro de las deformaciones con modelos Arima

Fuente: (Álvarez, 2018). 
Para determinar la estabilidad del modelo físico-numérico, utilizado en el proceso de modelación, así como la TEF seleccionada, se realizó un paso de calibración para garantizar la convergencia numérica en función de la reducción del coste computacional, tipo de elemento numérico, error asociado a la tecnología y tiempo de convergencia (véase figura 8).

En este paso se consideraron dos tipos de TEF, triángulos solo, cuadriláteros y triángulos, optando por la TEF, que presentó el menor error alcanzado, en referencia a la norma del valor real medido y normalizado por el valor patrón de menor error de desplazamiento numérico, alcanzado en las diferentes corridas de los modelos. Es por ello la selección del menor error posible bajo el criterio del menor costo computacional o tiempo de procesamiento de los datos, durante el proceso de convergencia numérica, lo cual permitió realizar una selección aceptable, acorde a un balance entre el mínimo posible de tiempo de convergencia y error probable, en el proceso de cálculo basado en las experiencias de Álvarez, Mesa y Recarey (2012).

Es por ello que, para abordar el proceso de las deformaciones en la estructura, se optó por la combinación de cuadriláteros y triángulos, con un orden de integración de cuarto orden y tercer orden respectivamente para cada elemento, y todos con nodos auxiliares, dado que al incrementar el orden de integración, se mantiene el error constante y solo se aumenta el costo computacional durante el proceso de solución del problema de contorno, a lo largo del dominio del medio continuo que se investiga, añadiendo un elemento de interface, como resultado de un refinamiento de la malla en las proximidades de los pilotes, en un valor relacional de 1/4 de unidad, a la dimensión de desratización del dominio o malla.

Por lo que se decidió realizar la modelación con la TEF Primera de Dimensión 1 m (DimTE1$1 \mathrm{~m})$, compuesta por cuadriláteros y triángulos, refinada en áreas próximas a los pilotes, a razón de $0.4 \mathrm{~m}$, con elementos de interface para aproximar lo mejor posible la respuesta del modelo al fenómeno de fricción entre los pilotes y el suelo (véase figura 8).

Figura 8. Representación gráfica del proceso de selección de la tecnología de elementos a emplear en el modelo físico-matemático

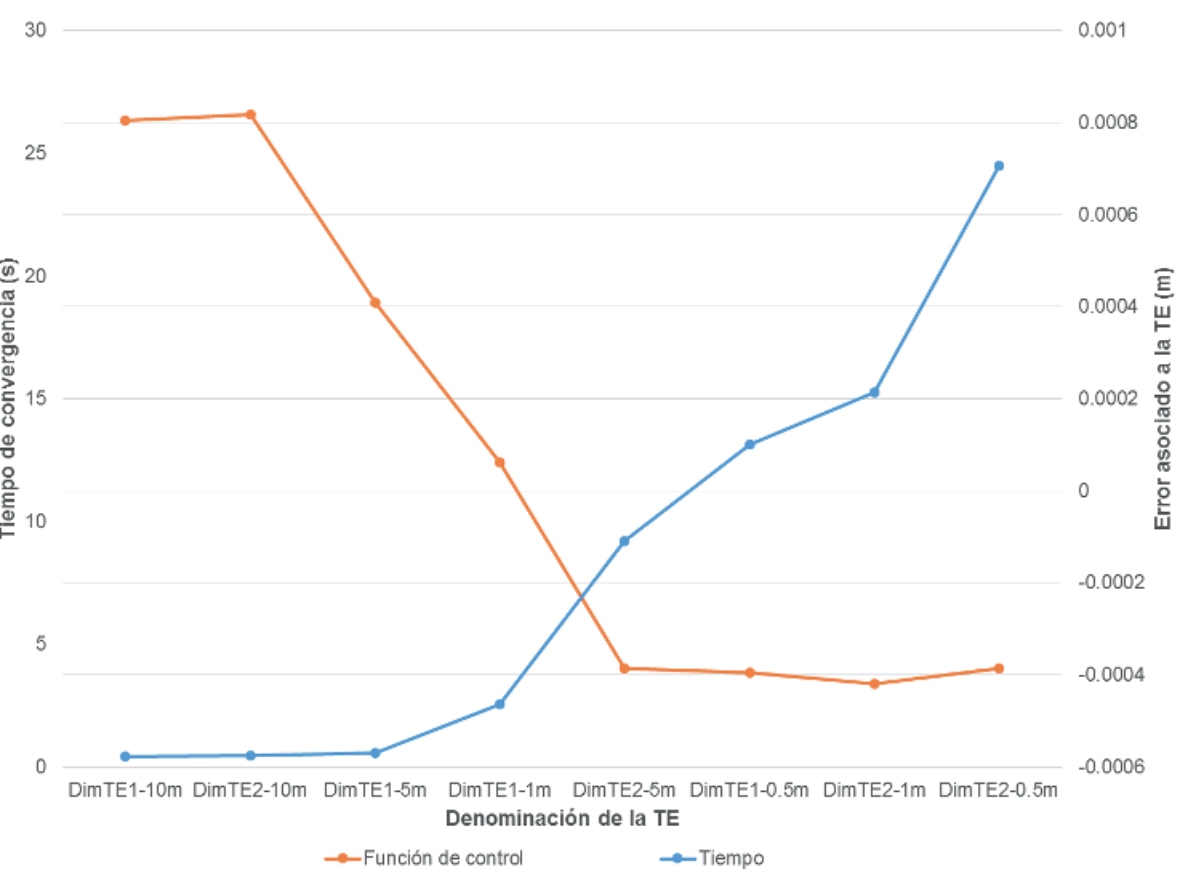




\section{Análisis de los resultados del modelo de Tensiones Iniciales (Tlo)}

En este acápite se analizó el comportamiento del estado inicial de las dos secciones longitudinales, por los ejes izquierdo y derecho de la estructura, para poder estimar inicialmente los parámetros esperados del comportamiento del material, acorde a los criterios de diseño inicialmente asumidos para la estructura, así como el comportamiento de las tensiones iniciales a las que el material se encuentra sometido por condición de peso propio, descartando todo criterio de posible acción o interacción por factores externos, según las condiciones iniciales impuestas al modelo (véase la figura 9).

Figura 9. Representación gráfica de las condiciones iniciales del modelo Tlo

\section{Sección longitudinal Eje Izquierdo}

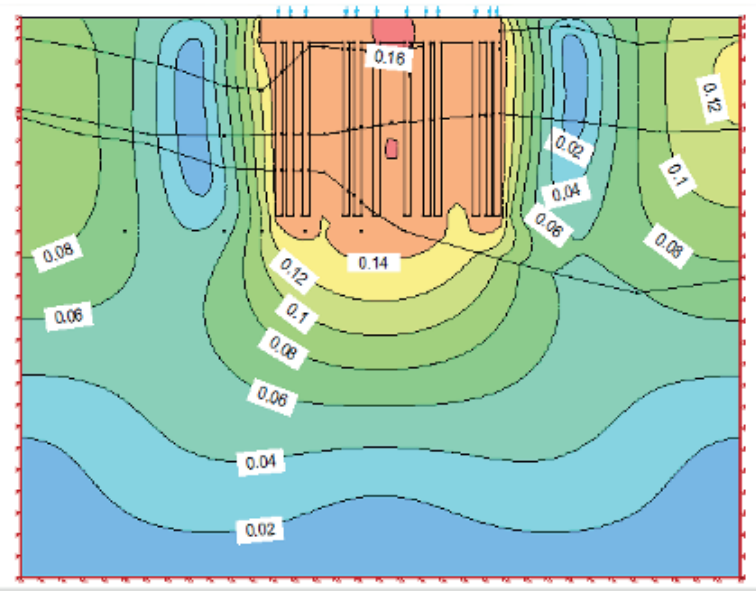

Sección longitudinal Eje Derecho

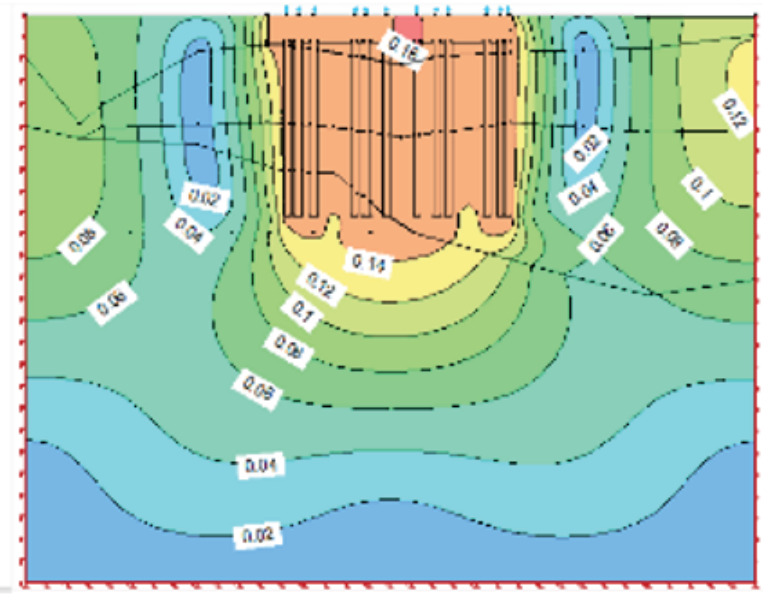

\section{Leyenda}

If Condición de borde con restricción en el movimiento horizontal.

Condición de borde con restricción de desplazamiento en las componentes vertical y horizontal.

En un primer instante se verificó el comportamiento del embudo de presiones, ejercido por la estructura bajo el principio de peso propio y como un criterio valorativo, según las normas norteamericanas para la mecánica de suelo y el diseño de cimentaciones profundas (Jones, 1986a, 1986b), y otros criterios similares contenidos en varias investigaciones (Craig, 1997; Naranbat, 2009).

Al evaluar visualmente el embudo de presiones bajo la estructura, se confirma que las características físico-geométricas, asumidas en el modelo, fueron correctas, pues se identificó el cierre del embudo de presiones con la relación de la dimensión del dominio y su discretización, conforme al estudio comentado en el acápite previo.

Al realizar un breve análisis de la respuesta de la superficie que se obtuvo en la figura 10, se identificó que fueron tomados nodos externos por fuera de la losa objeto de estudio, para visualizar el inicio y fin de la misma, la cual está identificada a partir del cambio de concavidad brusco, que realiza la superficie creada entre el borde de línea de los ejes izquierdo y derecho.

Además, se decidió realizar una verificación de la superficie creada entre los dos perfiles longitudinales, para evaluar si existe algún proceso de torsión en la losa, a partir de un posible 
trabajo diferencial de los pilotes, en el proceso de disipación de las cargas a las cuales está sometida la estructura, razón por la cual, al visualizar de forma ortogonal la superficie desde una perspectiva frontal, se identificó una zona donde se esperó aparecieran las deformaciones máximas en el centro cercano a los pilotes E, F y G del eje derecho de la losa, según el perfil longitudinal asumido en la presente investigación.

Figura 10. Representación gráfica de los desplazamientos esperados, resultantes del modelo $\mathrm{TI}_{0}$ vista en perspectiva

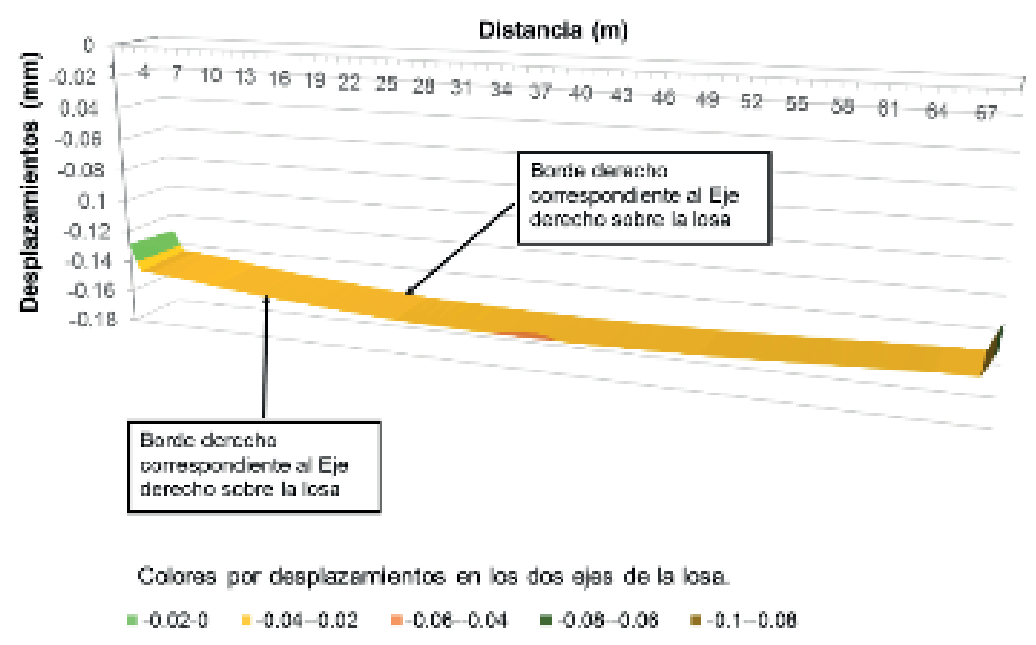

\section{Análisis de los resultados del modelo de Carga Deformación (C/D)}

En este paso fueron impuestas las cargas concéntricas sobre los centros geométricos de los pilotes, espacialmente distribuidas, acorde a la distribución de las masas sobre la losa, con base en que los datos máximos de deformaciones esperados ya se conocían, y han sido caracterizados y medidos por técnicas de la geodesia, con la adición de las tensiones iniciales del modelo Tlo al final del cálculo, para arribar teóricamente al estado tenso-deformacional actual en el que se encuentra la estructura.

En esta etapa se procedió a verificar el comportamiento del embudo de presión, ejercido bajo la losa y en los entornos cercanos a los pilotes, según se representa en la figura 11; y se coincide, prácticamente, con los datos estimados de manera previa en el modelo Tlo, por lo que, al no haber valores anómalos en las curvas de presión estimada por el modelo C/D, se constata que los pilotes están trabajando correctamente a fricción, para transmitir de forma homogénea las cargas soportadas por la losa al suelo.

Luego de haber comprobado el correcto funcionamiento de los pilotes, se realizó una verificación al comportamiento geométrico de la losa, dado que se espera una deformación máxima en el centro de la misma, próxima al extremo derecho, según se estableció como sistema de referencia en el modelo geométrico utilizado en el presente diagnóstico.

Al comparar los resultados alcanzados durante el proceso de modelación para la realización del diagnóstico de los estados de deformaciones de la losa, se decidió representar el comportamiento de los desplazamientos, como se muestra en la figura 12, la cual registra valores de los desplazamientos máximos para 16 mm, resultantes en el modelo C/D similar al Tlo. 
Figura 11. Representación gráfica de las condiciones finales del modelo $C / D$

\section{Sección longitudinal Eje Izquierdo}

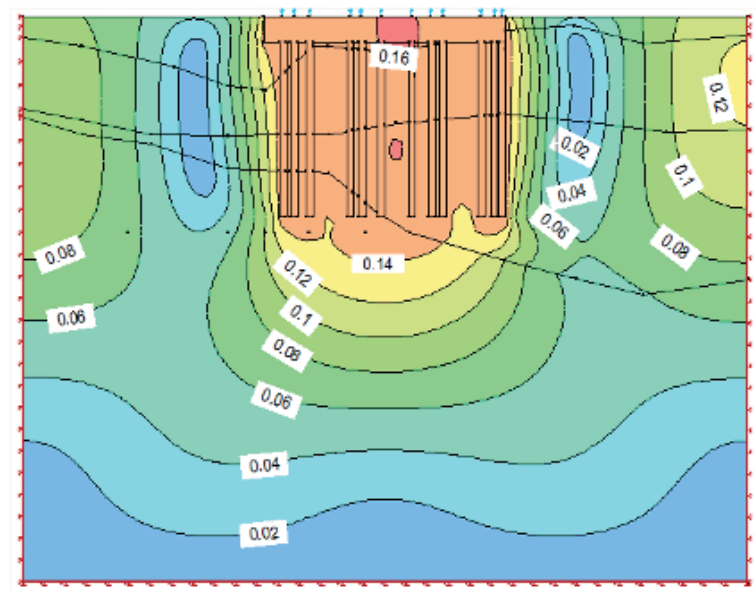

\section{Sección longitudinal Eje Derecho}

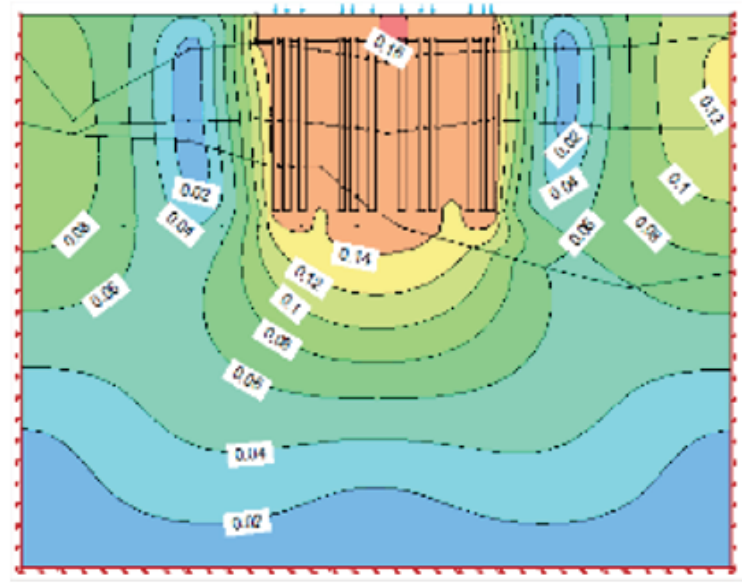

Este aspecto permitió corroborar que la losa trabaja de manera uniforme, a pesar de que la zona de torsión, esperada en los seis primeros metros, se localizó como punto medio alrededor de los $11.36 \mathrm{~m}$, desplazándose aproximadamente unos $2 \mathrm{~mm}$ más, respecto a la media de las deformaciones circundantes, con una leve inclinación hacia la derecha, según la distribución de masas aplicada sobre la estructura y por encontrarse el turbo generador eléctrico en esta zona.

A fin de evaluar el comportamiento de las diferencias de los desplazamientos registrados en los ejes izquierdo y derecho, se decidió representar ambos perfiles longitudinales, contrastados entre sí para poder verificar, en función de la distancia, si existen desplazamientos diferenciales que puedan conducir a una posible falla, según se muestra en la figura 12 , en la cual se muestra las diferencias que se obtuvo, las cuales no superan los $0.002 \mathrm{~m}$ en los puntos de mayor diferencia de cota entre los perfiles. Este aspecto implica que la losa soporta las cargas y manifiesta los desplazamientos registrados por métodos geodésicos de control de las deformaciones, sin que se identifique daños estructurales.

Figura 12. Representación gráfica de los desplazamientos en la losa por los perfiles longitudinales de los ejes izquierdo y derecho

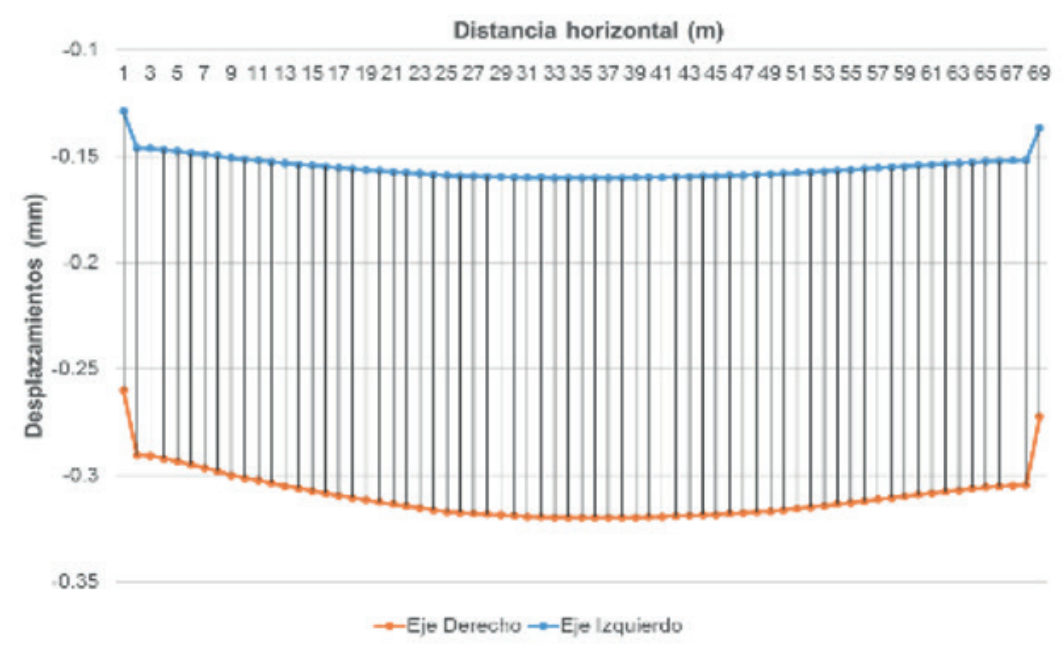


Además, al comparar los datos obtenidos, según los valores técnicos medidos en la Red Geodésica de Vigilancia y Control Técnico de las Deformaciones, se identificó que las diferencias del modelo C/D no superan los $0.002 \mathrm{~m}$, respecto a los datos medidos en campo, lo cual permite establecer márgenes de confiabilidad real entre los modelos teóricos y las mediciones de campo, para el presente diagnóstico.

\section{Pronóstico de las deformaciones}

Para el pronóstico de las deformaciones se consideraron fundamentalmente las experiencias publicadas por varios autores (Álvarez, 2018; Álvarez, Álvarez \& Vázquez, 2017; Feigl et al., 2020), en el uso de modelos Arima, y luego de obtener una serie de mediciones de los desplazamientos geodésicos, como la respuesta de la deformación en el suelo o datos térmicos, hidrológicos y mecánicos registrados en la superficie terrestre. Es por ello que, para el pronóstico de las deformaciones, se consideraron los datos iniciales medidos en cada uno de los ciclos, según se muestra en la tabla 3.

Tabla 3. Ejemplo de los datos utilizados para el cálculo de los modelos de pronóstico

\begin{tabular}{|c|c|c|c|c|c|c|c|}
\hline Ciclo & Fecha del ciclo & $\begin{array}{c}\text { Meses } \\
\text { acumulados }\end{array}$ & Días & $\begin{array}{c}\mathbf{H}^{\prime} \mathbf{1}^{\mathbf{2}} \\
\mathbf{( m m})\end{array}$ & $\begin{array}{c}\mathbf{H}^{\prime} \mathbf{1 - 2} \\
\mathbf{( m m})\end{array}$ & $\begin{array}{c}\text { H1 } \\
\mathbf{( m m})\end{array}$ & $\begin{array}{c}\text { H1-2 } \\
\mathbf{( m m})\end{array}$ \\
\hline 1 & $12 / 10 / 1983$ & 0 & 0 & 0 & 0 & 0 & 0 \\
\hline 2 & $12 / 11 / 1985$ & 23 & 8395 & -4.6 & -4.9 & -5.0 & -5.2 \\
\hline 3 & $24 / 11 / 1987$ & 46 & 16790 & -10.9 & -11.4 & -12.0 & -12.1 \\
\hline 4 & $12 / 11 / 1988$ & 58 & 21170 & -15.7 & -16.3 & -17.0 & -17.3 \\
\hline 5 & $20 / 10 / 1989$ & 69 & 25185 & -17.5 & -18.0 & -18.9 & -19.2 \\
\hline 6 & $06 / 12 / 1990$ & 83 & 30295 & -19.1 & -19.8 & -20.5 & -21.4 \\
\hline 7 & $10 / 05 / 1991$ & 88 & 32120 & -17.5 & -18.6 & -19.3 & -19.8 \\
\hline 8 & $10 / 04 / 1992$ & 99 & 36135 & -17.5 & -18.7 & -19.3 & -19.8 \\
\hline 9 & $20 / 11 / 2007$ & 286 & 104390 & -55.1 & -56.9 & -59.0 & -60.8 \\
\hline 10 & $06 / 08 / 2009$ & 307 & 112055 & -55.6 & -57.3 & -58.9 & -60.9 \\
\hline 11 & $15 / 05 / 2010$ & 316 & 115340 & -56.3 & -58.0 & -59.9 & -61.9 \\
\hline 12 & $29 / 06 / 2011$ & 329 & 120085 & -56.7 & -58.3 & -60.7 & -62.6 \\
\hline 13 & $25 / 04 / 2014$ & 364 & 132860 & -58.8 & -60.5 & -62.5 & -64.9 \\
\hline 14 & $19 / 12 / 2015$ & 384 & 140160 & -59.7 & -61.6 & -63.6 & -65.7 \\
\hline
\end{tabular}

Para poder utilizar la teoría de series cronológicas (Box, 2015; Box \& Jenkins, 1970), fue necesario realizar una interpolación de datos por medio de un spline bicúbico (consultar la figura 13). Este proceso facilitó la obtención de una serie de datos continuos ordenados cronológicamente, según se muestra en la figura 14, en la que se detalla el proceso de interpolación, durante los 187 meses en los que no fueron realizadas mediciones.

Además, es necesario aclarar que, los pronósticos realizados fueron en consideración a 5 años, posteriores a la fecha del último ciclo, con una ventana de control de 1 año de solape,

5 Los datos que contienen el símbolo prima Ej.: H'1, corresponden al eje izquierdo y los que no poseen este símbolo corresponden al eje derecho. 
para verificar el posible error entre el valor pronosticado por el modelo y los datos medidos e interpolados (ver tabla 4).

Figura 13. Representación gráfica del proceso de interpolación de datos y su control numérico
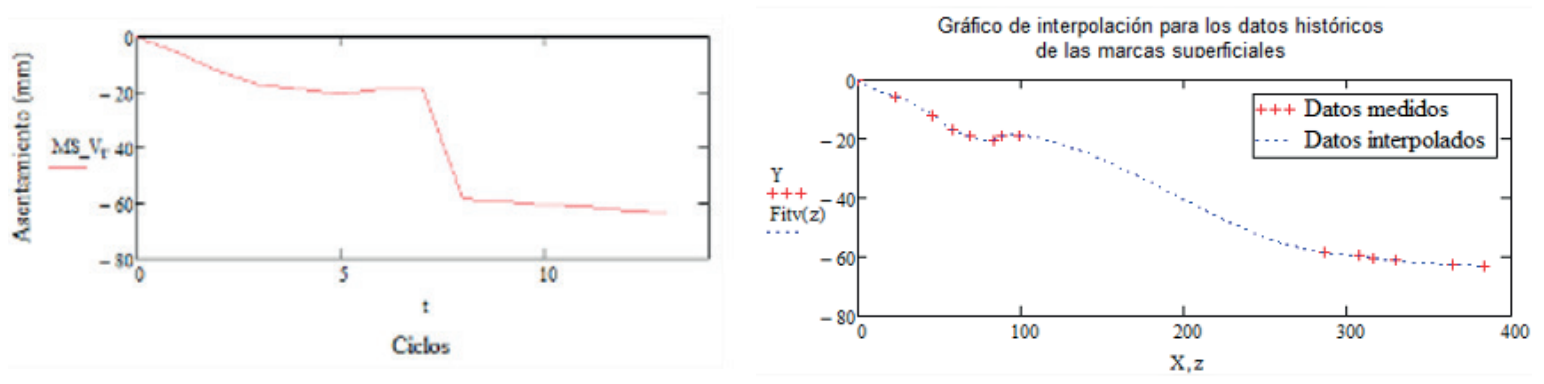

Gráico de control a la interpolación para los datos históricos de las marcas subericiales

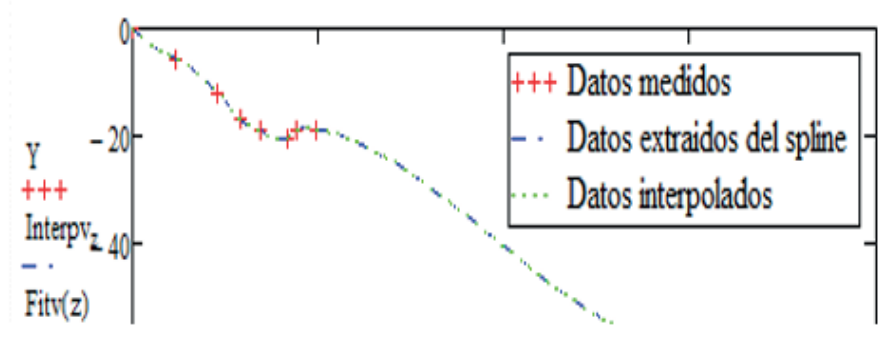

Figura 14. Representación gráfica del modelo de pronóstico, con los intervalos de confianza para la marca H'1

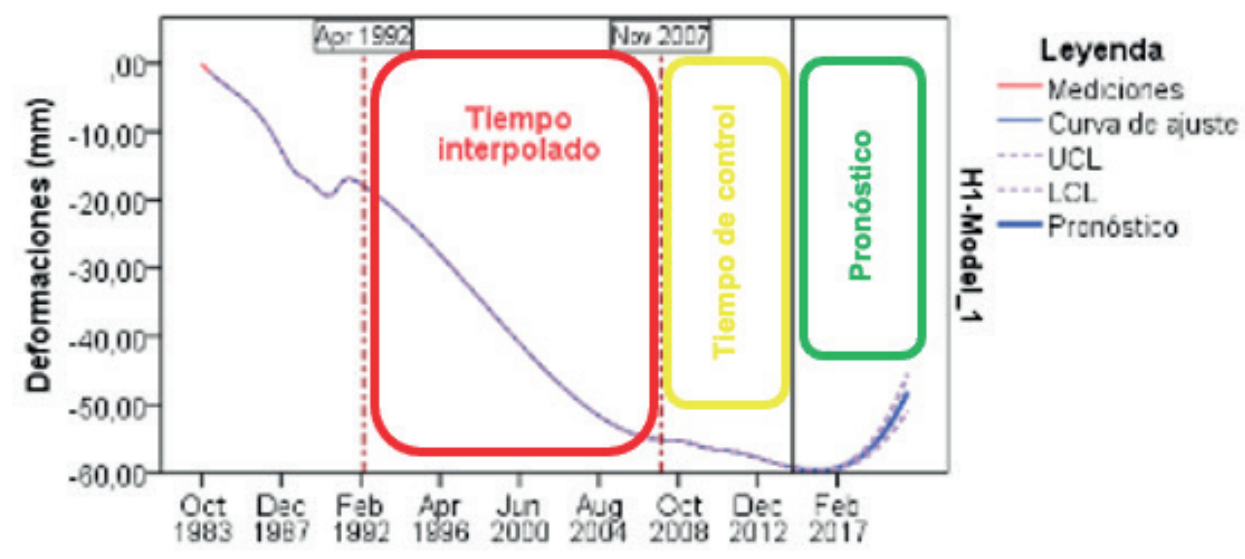

Tiempo (meses/año) 
Tabla 4. Cálculo del error obtenido en el pronóstico, respecto a los datos reales medidos e interpolados para la marca superficial $\mathrm{H}^{\prime} 1$

\begin{tabular}{|c|c|c|c|}
\hline Fecha (mes/año) & Pronóstico $(\mathbf{m m})$ & $\begin{array}{c}\text { Dato real e interpolado } \\
(\mathbf{m m})\end{array}$ & Diferencias $(\mathbf{m m})$ \\
\hline Oct. 2014 & -59.2792 & -59.2792 & 0.0000 \\
\hline Nov. 2014 & -59.3302 & -59.3302 & 0.0000 \\
\hline Dic. 2014 & -59.3787 & -59.3787 & 0.0000 \\
\hline Ene. 2015 & -59.4246 & -59.4246 & 0.0000 \\
\hline Feb. 2015 & -59.4678 & -59.4679 & 0.0000 \\
\hline Mar. 2015 & -59.5082 & -59.5082 & 0.0001 \\
\hline Abr. 2015 & -59.5456 & -59.5457 & 0.0001 \\
\hline May. 2015 & -59.5798 & -59.5800 & 0.0001 \\
\hline Jun. 2015 & -59.6108 & -59.6110 & 0.0002 \\
\hline Jul. 2015 & -59.6384 & -59.6387 & 0.0003 \\
\hline Aug. 2015 & -59.6624 & -59.6628 & 0.0004 \\
\hline Sep. 2015 & -59.6828 & -59.6833 & 0.0005 \\
\hline Oct. 2015 & -59.6993 & $-59.7000^{6}$ & 0.0007 \\
\hline
\end{tabular}

Como se muestra en la tabla 4, los errores asociados al modelo, en el año de control establecido para la serie cronológica, presentaron diferencias insignificantes, consideradas como errores blancos, por lo que fue factible el pronóstico realizado para el periodo de 5 años, si se considera que las condiciones tenso-deformacionales del modelo, acorde al estado actual de solicitación de la losa, no varíen.

Para poder inspeccionar si la losa posee un comportamiento uniforme, se representó todos los desplazamientos en un gráfico cronológico, según se muestra en la figura 15. En este caso, se evidencia una leve dispersión de los desplazamientos en el último ciclo, por lo que implica posibles comportamientos generados, a partir de la no coincidencia de la geometría del centro de masa, de las cargas colocadas sobre la losa, respecto a la distribución de los pilotes.

Figura 15. Representación gráfica del comportamiento de los desplazamientos de la losa
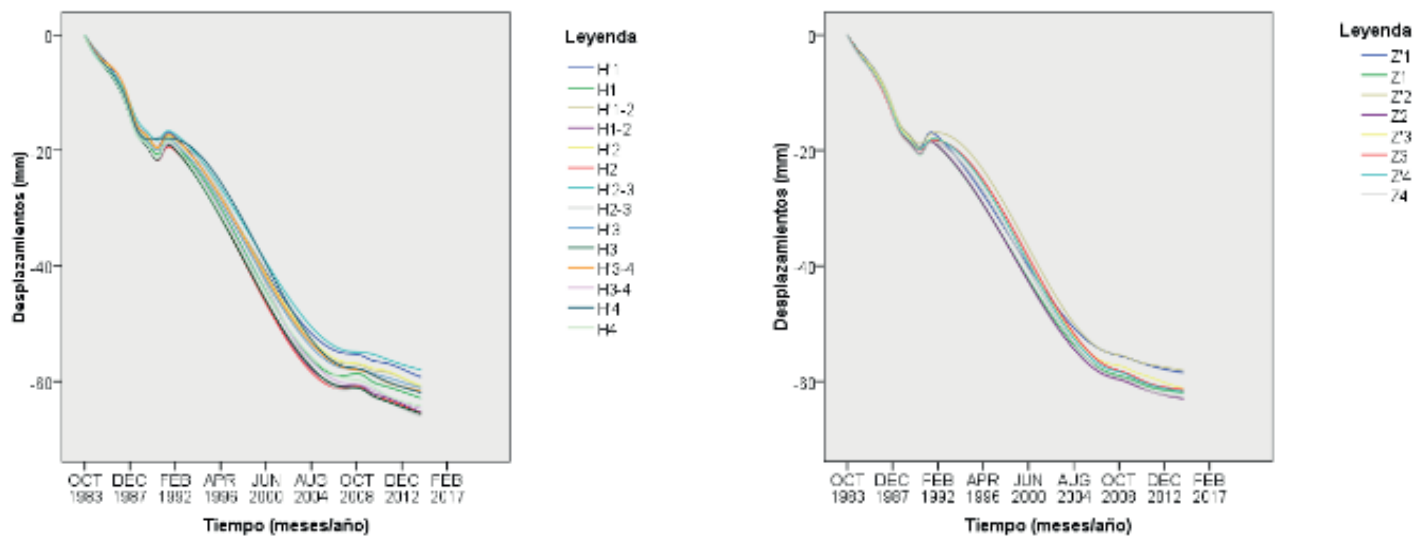

$6 \quad$ Este dato corresponde a una medición real realizada por el Grupo Empresarial de Geocuba VC-SS; el resto, contenido en esta columna, son datos interpolados por el método del spline bicúbico, con un estricto control de los errores numéricos y su control de propagación de errores. 
A continuación, se realiza un ejemplo completo con los resultados de obtención y pronóstico del modelo para la marca H'1 (ver tabla 5, tabla 6 y figura 16).

Tabla. 5. Datos generales del modelo físico-matemático-estadístico

\begin{tabular}{|c|c|c|c|}
\hline \multicolumn{3}{|c|}{ Descripción del modelo } & Tipo de modelo \\
\hline Modelo ID & $\mathrm{H}^{\prime} 1$ & Model_1 & Arima $(3,5,3)(0,0,0)$ \\
\hline
\end{tabular}

\begin{tabular}{|c|c|c|c|c|c|c|}
\hline \multicolumn{7}{|c|}{ Estadística del modelo } \\
\hline \multirow{2}{*}{ Modelo } & \multirow{2}{*}{$\begin{array}{l}\text { Cantidad de } \\
\text { predictores }\end{array}$} & \multirow{2}{*}{$\begin{array}{c}\begin{array}{c}\text { Est. del ajuste } \\
\text { del modelo }\end{array} \\
\text { R-cuadrado } \\
\text { estacionario }\end{array}$} & \multicolumn{3}{|c|}{ Ljung-Box Q(18) } & \multirow{2}{*}{$\begin{array}{l}\text { Cantidad de } \\
\text { pronósticos }\end{array}$} \\
\hline & & & Estadística & DF & Sig. & \\
\hline H'1-Modelo_1 & 1 & 1.000 & 51.364 & 12 & 0.000 & 69 \\
\hline
\end{tabular}

Figura 16. Representación gráfica del pronóstico de los desplazamientos para la marca superficial $H^{\prime} 1$

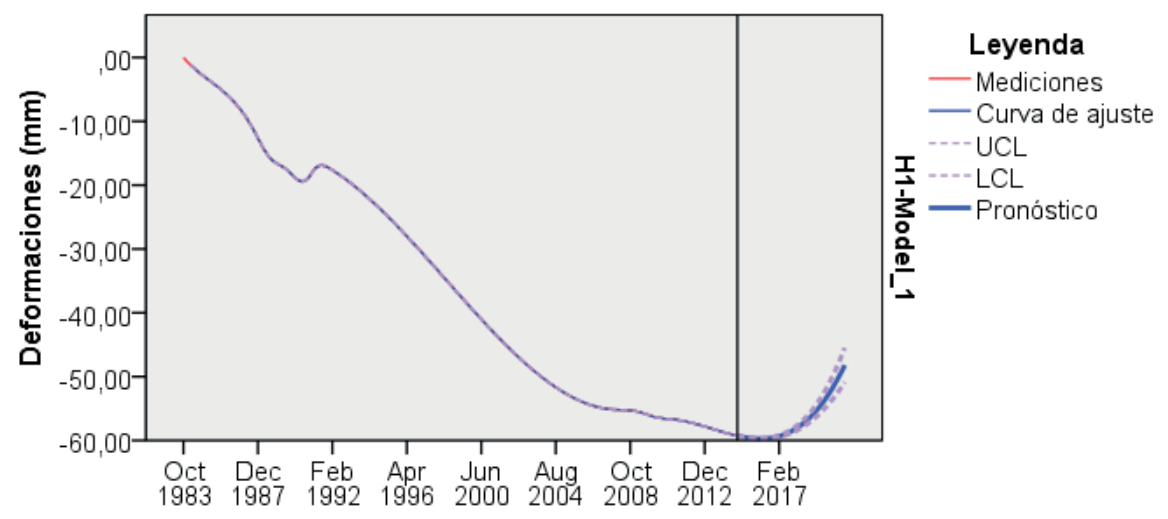

Tiempo (meses/año)

Tabla 6. Ejemplo de los resultados alcanzados en el pronóstico de la marca superficial H'1

\begin{tabular}{|c|c|c|c|c|c|c|c|}
\hline \multicolumn{2}{|c|}{ Modelo } & Nov. 2018 & Dic. 2018 & Ene. 2019 & $\ldots$ & Sep. 2020 & Oct. 2020 \\
\hline \multirow{3}{*}{ H'1-Modelo_1 } & Pronóstico & -56.3132 & -56.0774 & -55.8320 & $\ldots$ & -48.6812 & -48.1994 \\
\cline { 2 - 8 } & ICS & -55.5858 & -55.2988 & -54.9998 & $\ldots$ & -46.1215 & -45.5143 \\
\cline { 2 - 8 } & ICI & -57.0405 & -56.8559 & -56.6642 & $\ldots$ & -51.2409 & -50.8845 \\
\hline
\end{tabular}

Similar proceso fue realizado para todas las marcas superficiales, de las cuales solo serán presentadas las gráficas de pronósticos finales (figura 17), omitiendo todo el tecnicismo matemático-estadístico implementado de similar rigurosidad en el proceso de cálculo y estimación de datos. 
Figura 17. Representación gráfica del pronóstico de los desplazamientos para la marca superficial H1

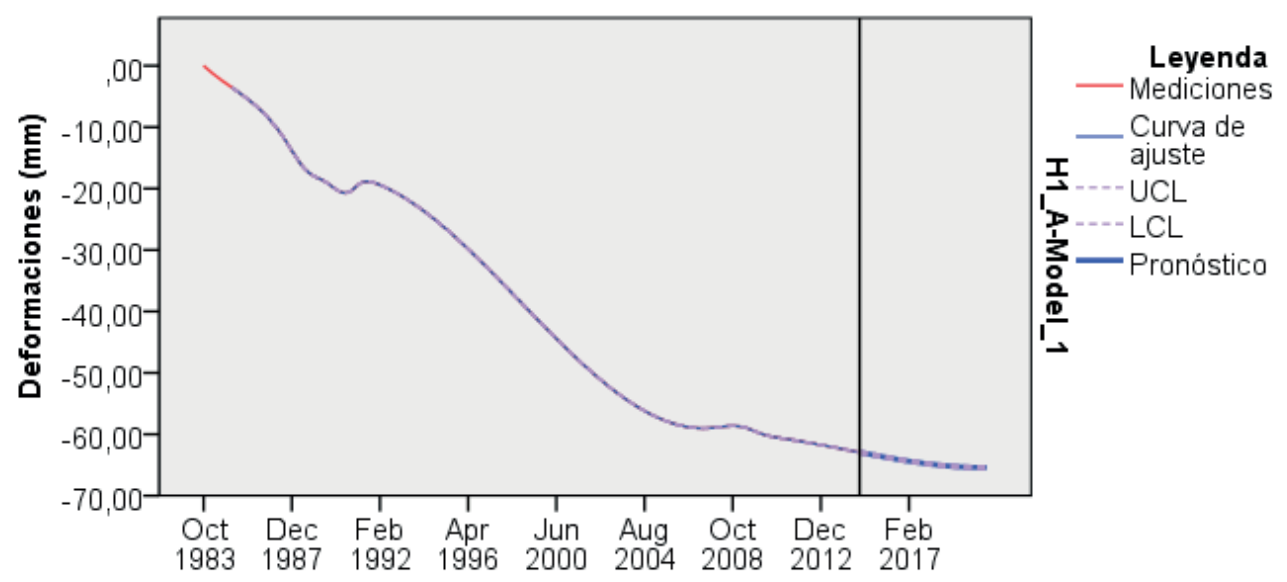

Tiempo (meses/año)

\section{Conclusiones y recomendaciones}

Acorde a los resultados alcanzados, se arribaron a las siguientes conclusiones:

- Al comparar los resultados teóricos, obtenidos en función del comportamiento de los niveles de surgencia de la LCS teórica después de la colocación del filtro, en contraste a los estudios previos realizados, se obtuvo resultados superiores a $-1.00 \mathrm{~m}$ de disminución del nivel freático teórico registrado en las diferentes calas de control y piezómetros.

- En las modelaciones de los diferentes filtros, se alcanza una reducción del caudal, que pasa por la estructura de la cortina y la base de cimentación. Estos datos fueron comparados con los medidos delante del filtro y los valores teóricos alcanzados en los modelos virtuales, después de la colocación de las variantes de solución. Los resultados obtenidos fueron múltiples valores de gastos, con una diferencia no mayor de $2.5 \mathrm{E}-06 \mathrm{~m} 3 / \mathrm{s}$, como se evidencia en el proceso de redireccionamiento del flujo en el interior de la cortina, por el correcto funcionamiento de la propuesta de los nuevos filtros, expresado en términos de amenaza, vulnerabilidad y riesgo.

- Al realizar el análisis de la estabilidad de taludes para los diferentes escenarios, se obtienen resultados que superan el valor normativo como criterio mínimo para el Factor de Seguridad del talud, por lo que se ratifica como válida la solución de ingeniería empleada en el talud, aguas abajo de la cortina en el embalse Zaza. Se recomienda la construcción del filtro de tipo prisma, por ser muy fácil su ejecución constructiva, por parte de las empresas constructoras especializadas en trabajos hidráulicos a nivel nacional. 


\section{Referencias}

Álvarez, J., Mesa, M. y Recarey, C. (2012). Técnicas de calibración numérica para problemas de ingeniería. Ciudad de La Habana, Cuba: Departamento de Ingeniería Civil. Instituto Superior Politécnico José Antonio Echeverría (Ispjae) de La Habana, Centro de Investigación de Mecánica Computacional y Métodos Numéricos en la Ingeniería (CIMCNI), de la Universidad Central Marta Abreu de Las Villas.

Álvarez, L. (1998). La estabilidad de cortinas de presas de tierra mediante la solución de los estados tenso-deformacionales y la aplicación de la teoría de seguridad (tesis presentada en opción al Grado Científico de Doctor en Ciencias Técnicas). Universidad Central Marta Abreu de Las Villas, Santa Clara.

Álvarez, M. (2018). Pronóstico de deformaciones en cortinas de presas de tierra (tesis presentada en opción al Grado Científico de Doctor en Ciencias Técnicas). Universidad Central Marta Abreu de Las Villas, Instituto Superior Politécnico José Antonio Echeverría (Ispjae) de La Habana, Centro de Investigación de Mecánica Computacional y Métodos Numéricos en la Ingeniería (Cimcni), Universidad Central Marta Abreu de Las Villas, La Habana, Cuba.

Álvarez, M., Álvarez, L. y Vázquez, R. (2017). Risk Assessment and Modeling of Technical Solutions for Filtrations Earth Dams. Enfoque UTE, 8(5), pp. 26 - 36. doi: 10.29019/enfoqueute.v8n5.177

Blanco, E. E. E., Pacheco, C. E. Á. \& Moreno, G. G. (2020). Modelamiento del tambor de una caldera acuotubular para una planta de energía térmica. Revista colombiana de tecnologias de avanzada (RCTA), 1(35). doi: 10.24054/16927257.v35.n35.2020.3924

Box, G. y Jenkins, G. (1970). Time Series Analisys Forecasting and Control. Holden-Day.

Box, G., Jenkins, G., Reinsel, G. y Ljung, G. (2015). Time Series Analysis: Forecasting and Control (5. ${ }^{\circ}$ ed.). Wiley.

Craig, R. (1997). Soil Mechanics (6. ${ }^{\circ}$ ed.). CRC Press

Delgado, P. (2011). Sistema auscultación de obras subterráneas. ACRE Surveying Solution.

Feigl, K. L., Reinisch, E. C., Batzli, S. A. et al. (2020). Spatio-Temporal Analysis of Deformation at San Emidio Geothermal Field, Nevada, USA between 1992 and 2010. Remote Sensing, 11(16), 1935. doi: $10.3390 /$ rs 11161935

Hernández, F. (2012). Asentamientos permanentes por sismo en presas de tierra y enrocamiento. El posgrado en la Ingeniería Civil; Il Encuentro. https://bit.ly/2H1kGqD

Jones, J. (1986a). Foundations and Earth Structures Design Manual 7.02 (DM-7.02). Alexandria, Virginia, United States of America: Naval Facilities Engineering Command (NAVFAC).

Jones, J. (1986b). Soil Mechanics Design Manual 7.01 (DM-7.01). Alexandria, Virginia, United States of America: Naval Facilities Engineering Command (NAVFAC).

Kopáčik, A., Erdélyi, J. y Kyrinovič, P. (2020a). Design, Construction, and Operation of Nuclear Power Plants: Geodetic Activities. In A. Kopáčik, J. Erdélyi, \& P. Kyrinovič (Eds.), Engineering Surveys for Industry (pp. 197-213). Springer International Publishing. doi: 10.1007/978-3-030-48309-8_10

Kopáčik, A., Erdélyi, J. y Kyrinovič, P. (2020b). Setting-Out and Measurement of Cranes and Crane Runways. Engineering Surveys for Industry (pp. 143-178): Springer.

Naranbat. (2009). Nociones de Geología e Ingeniería Geológica para Ingenieros. s.l.f.

Quevedo, G. (2001a). Análisis de la estabilidad de taludes con la aplicación del método de las diferencias finitas. Santa Clara, Villa Clara, Cuba: Universidad Central Marta Abreu de Las Villas, Facultad de Construcciones.

Quevedo, G. (2001b). Estudio de la estabilidad de taludes y laderas para la prevención de desastres. Santa Clara, Villa Clara, Cuba.

Recarey, C. (1999). Modelación del terreno y las estructuras en el dominio del tiempo (tesis presentada en opción al Grado Científico de Doctor en Ciencias Técnicas). Facultad de Construcciones, Universidad Central Marta Abreu de Las Villas, Santa Clara, Villa Clara, Cuba. 